\title{
New Construction of an Electronic Nose Detects Volatile Organic Compounds from Blood, useful for the Diagnosis and Screening of Ovarian Carcinoma
}

\section{Gyorgy Horvath $^{1 *}$, Jonas Ranstam ${ }^{2}$, Mattias Ottosson ${ }^{3}$ and Morgan Nilsen ${ }^{3}$}

${ }^{1} /$ nstitute of Clinical Sciences, University of Gothenburg, Gothenburg, Sweden

${ }^{2}$ Department of Clinical Sciences, Orthopaedics, Lund University, Lund, Sweden

${ }^{3}$ University West Sweden, Trollhättan, Sweden

\begin{abstract}
Objective: Ovarian cancer is the fifth-most common cause of cancer-related death in women. As a result of these high mortality rates, ovarian cancer fulfils some of the criteria necessary for early detection and for introduction of population screening: It is an important health problem, and early detection is associated with improved outcomes. However, there are no safe methods for early diagnosis nor any accepted screening programmed for ovarian cancer. Experimental data indicated that the detection of volatile organic compounds released by various cancer cells may be useful in diagnosing cancer.
\end{abstract}

Methods: We constructed an electronic nose that is adapted in a number of ways to analyses volatile organic compounds (VOCs) from blood.

Results: A statistical analysis of the detected signals from 165 patients showed an AUC 95\% with $\mathrm{Cl}$ of $0.9559-$ 0.9929. The sensitivity was $92 \%$ and the specificity was $93 \%$.

Conclusion: This device is the first clinically useful tool for the early diagnosis of ovarian carcinoma. The operation of the instrument is based on the accurate detection of specific VOC: $\mathrm{s}$ from blood that are characteristic of ovarian carcinoma.

Keywords: Development; Electronic nose; Ovarian cancer; VOCs; Early diagnosis; Population screening

Abbreviations: VOC: Volatile Organic Compounds; FIGO: International Federation of Gynaecology and Obstetrics; AUC: Area Under the Curve; ROC: Receiver Operating Characteristic; URMS: Uniform Requirements for Manuscripts Submitted to Biomedical Journals

\section{Introduction}

Worldwide, there are more than 234,000 new cases of ovarian cancer annually, accounting for approximately $4 \%$ of all cancers diagnosed in women. Incidence rates vary considerably, with the highest rates in the USA and Northern Europe and the lowest rates in Africa and Asia. Approximately 43,000 cases occur each year in Europe, and 22,000 cases occur each year in the USA. In the EU, the lowest rates are in the Southern European countries of Greece, Portugal and Cyprus, while the highest rates are in the Northern and Eastern European countries of Lithuania, Denmark, the Czech Republic and Estonia. The five-yearsurvival of patients is approximately $48 \%$ worldwide, and there are more than 100,000 deaths each year.

In Sweden, the disease represents $3.1 \%$ of all cancer cases in women, with approximately 700 cases per year. Despite a relatively low incidence rate, ovarian cancer is the fifth-most common cause of cancer death in women.

As a result of these high mortality rates, ovarian cancer fulfils some of the criteria necessary for the introduction of population screening: it is an important health problem, and early detection is associated with improved outcomes. Potential screening tests for ovarian cancer have not yet been shown to reduce mortality, although both ultrasound and tumour markers can detect a significant proportion of ovarian cancers [1]. Currently, there is no accepted screening programme for ovarian cancer.
In a previous study [2], we clearly demonstrated that human ovarian carcinomas can be characterized by a specific odour (ovarian cancer-specific volatile organic compounds, VOCs) detectable by a trained dog. That study also showed that a dog can be trained to distinguish between different histopathological types and grades of ovarian carcinomas, including borderline tumours, as well as healthy control samples. Double-blind tests showed $100 \%$ sensitivity and $97.5 \%$ specificity. Moreover, the VOCs of ovarian carcinomas appeared to differ from those of other gynaecological malignancies such as cervical, endometrial, and vulvar carcinomas, suggesting different VOC characteristics for different malignancies. The study further showed that early-stage and low-grade ovarian carcinomas emitted the same specific smell as did advanced tumours. Although the dog did not indicate healthy postmenopausal ovary samples as targets, we believe that the carcinoma-specific VOCs were organ-dependent, at least to some extent.

The results of later studies $[3,4]$ that we published showed that trained dogs could distinguish between blood from patients with various types of ovarian carcinoma and blood from healthy individuals. The studies demonstrated clearly that the same VOCs were emitted by the blood as by the ovarian carcinoma samples. Moreover, the dogs were able to identify a drop of blood plasma from patients with ovarian carcinoma

*Corresponding author: György Horvath, Institute of Clinical Sciences, University of Gothenburg, 41345 Gothenburg, Sweden, Tel: +46-857024620; E-mail gyorgyhorvath89@yahoo.se

Received October 22, 2018; Accepted December 13, 2018; Published December 15,2018

Citation: Horvath G, Ranstam J, Ottosson M, Nilsen M (2018) New Construction of an Electronic Nose Detects Volatile Organic Compounds from Blood, useful for the Diagnosis and Screening of Ovarian Carcinoma. J Cancer Sci Ther 10: 387-391. doi: 10.4172/1948-5956.1000572

Copyright: @ 2018 Horvath G, et al. This is an open-access article distributed under the terms of the Creative Commons Attribution License, which permits unrestricted use, distribution, and reproduction in any medium, provided the original author and source are credited. 
Citation: Horvath G, Ranstam J, Ottosson M, Nilsen M (2018) New Construction of an Electronic Nose Detects Volatile Organic Compounds from Blood, useful for the Diagnosis and Screening of Ovarian Carcinoma. J Cancer Sci Ther 10: 387-391. doi: 10.4172/1948-5956.1000572

that showed negative results by an established tumour marker, CA125. The relatively high concentration of VOC molecules in the blood suggests that the same volatile components may be detected in exhaled breath samples or the skin of patients. Moreover, the same studies showed that neither differences in grade or different stages of ovarian cancers affected the sensitivity and specificity suggests interesting properties for VOC based diagnostics [2-4]. To our knowledge no other authors published links between VOC diagnostics and tumor differentiation or different stages of the disease.

These results strongly suggested that specific VOCs emitted by various carcinomas may be useful for early diagnosis and differential diagnosis and for the screening of various malignant diseases in the future once it becomes technologically possible.

After our project with trained dogs that led to many interesting observations in addition to those we published as the basis for further work, we began working to develop an electronic nose capable of detecting and registering ovarian cancer-specific VOCs in the "headspace" of blood.

The aim of this study was to present only the process and the results after several years of development work, but not to conduct a full-scale clinical study on ovarian cancer diagnostics. The first results of a study with limited number of patients conducted with the new instrument will be presented here.

\section{Materials and Methods}

\section{Plasma isolation}

Blood samples were obtained before primary surgery from patients with ovarian carcinoma. These samples were collected in EDTA tubes and then centrifuged at $3000 \mathrm{rpm}$ for $10 \mathrm{~min}$. and with plasma pots over the small plastic tubes. After undergoing CA-125 analysis, the remaining plasma samples were kept at $-80^{\circ} \mathrm{C}$ in our tumour bank. Only samples from patients with histologically verified ovarian cancer were saved in the tumor bank. Control blood samples were collected from healthy female individuals between 30 and 50 years of age. During and after preparation, the plasma samples were treated identically as the target samples. For the present study, 97 plastic tubes with blood plasma from ovarian cancer patients were selected. Forty of these samples were from FIGO (the International Federation of Gynaecology and Obstetrics) Stage I-II patients, and 57 samples were from Stage IIIIV patients. Donor patients were between the ages of 30 and 70 -yearsold. The mean age of the healthy women in the control group was slightly lower than that of the patients with ovarian carcinoma. This choice was deliberate: by choosing a lower age of control individuals, we reduced the risk of including control patients not yet diagnosed ovarian cancer.

During sample selection, neither the tumour grade and the histopathological subtypes of ovarian carcinomas nor other clinical data were considered. The purpose of the study was to obtain a general picture of the possibility of using the method of ovarian cancer diagnostics, which explains why we did not consider most clinical and biological aspects in material selection. Clinical studies with detailed clinical and pathological reporting are currently under implementation.

In addition to the samples from ovarian cancer patients, 68 control samples were randomly selected. Thus, a total of 165 samples were analysed. Plasma samples from both cancer and control patients were marked only by code numbers, and neither clinical nor personal data were requested from the tumour bank. The selection of samples was carried out by laboratory staff who did not participate in either the analysis or in the evaluation.

\section{Gas chromatography}

The gas chromatography and mass spectrometry (GS + MS) was performed by Johan Redeby, Royal Institute of Technology, Stockholm, Sweden.

Extended gas chromatography and mass spectrometry (Agilent GC6890 with a mass detector for MS; Agilent Technologies, USA) were performed to show that there was a difference in the VOCs emitted by healthy and ovarian carcinoma tissues. A further object of the GS+MS analysis was to use the results to aid with the choice of sensors to be built into the planned electronic nose. The GS+MS analysis was, however, not intended as a scientific study, but only as a guide to the planned work (spot-check).

\section{Electronic nose}

Currently two prototypes of the new device are in use (Figure 1). The device was built on the well-known basic principles of an electronic nose, containing 32 gas sensors designed to detect the maximum number of VOCs emitted in plasma. Initially, our simple electronic nose was not capable of detecting VOCs signals. By successive steps, we first adapted a pipe system for the analysis of liquids, built new control cards, and improved the amplifiers of each sensor coupled to a potentiometer to individually regulate the "heating voltage". Between these steps, changes were tested by analysing and evaluating the results from 40-50 samples. The Labview software [5] was programmed to control the analytical processes, calculate statistics and monitor the safety of the unit's functions. Several hundred plasma samples were used in the learning processes of the statistical programmes and the device tests during construction. In addition to the 11 VOCs that are characteristic for ovarian cancer, the device itself and the method used for processing the individual signals from blood plasma are protected both by the Swedish Patent and Registration Office and by the Patent Cooperation Treaty (public).

\section{Usage}

The device is easy to use and is designed such that lab technicians can easily handle it. Before using the instrument, the sensors need to warm up for at least 1 hour. The analysis process is automated except for the input and removal of samples. For the input of sample material, a specially designed input rod with a place for the plasma is used. The input rod is a disposable item to ensure that the rod is odourless during use. For a complete analysis, only $0.8-1.0 \mathrm{ml}$ of plasma needed. After placing the samples into the instrument, a fan is automatically triggered, which draws air over samples and moves the VOC's from the sample headspace through the sensor tube. In the presence of a detectable VOC's, the individual sensor's conductivity increases depending on the VOC's concentration in the air. A built-in hardware (USB-6218, National Instruments, USA) convert the change in conductivity to an output signal (in Volt) which corresponds to the gas concentration. Meanwhile 0 - 360 seconds (active time) concentration of VOC's in the sensor tube increases by increasing amounts released from plasma. The process is recorded in the program as rising signal curves. The sample is taken out at 360 seconds, then signals from the so-called sensor recovery time (360-600 seconds) are recorded. Ten output signals in Volt are recorded from each sensor per second during analysis. The values are recorded in an Excel table, a total of 192000 values per analysis. These measured values from each individual sensor are automatically processed in an algorithm. Analysis results are clear 
Citation: Horvath G, Ranstam J, Ottosson M, Nilsen M (2018) New Construction of an Electronic Nose Detects Volatile Organic Compounds from Blood, useful for the Diagnosis and Screening of Ovarian Carcinoma. J Cancer Sci Ther 10: 387-391. doi: 10.4172/1948-5956.1000572

after 10 minutes. Assay results are reported as cancer or not cancer. After five minutes of resting, the next analysis may be performed. If there are no VOCs in the samples all 32 signal curves remain on the baseline and the final results are displayed as not-a-number $(\mathrm{NaN})$.

\section{Statistical analysis}

A classification (prediction) model was developed using logistic regression of the sensor readings during several specified time intervals with LASSO (least absolute shrinkage and selection operator), and with the degree of gradient or of shrinkage specified using cross-validation [6] in order to avoid overfitting. The accuracy of the developed classification model was estimated using ROC (receiver operating characteristic) curves, and the optimal sensitivity and specificity were derived from the ROC curve.

The developed classification model was then programmed into Lab view, which is an integrated part of the device, for predictions for individual patients.

The statistical calculations for the development of the classification model were performed using $\mathrm{R}$ ver. 2.3.2 [7] and the packages glmnet [8], caret [9] and pROC [10].

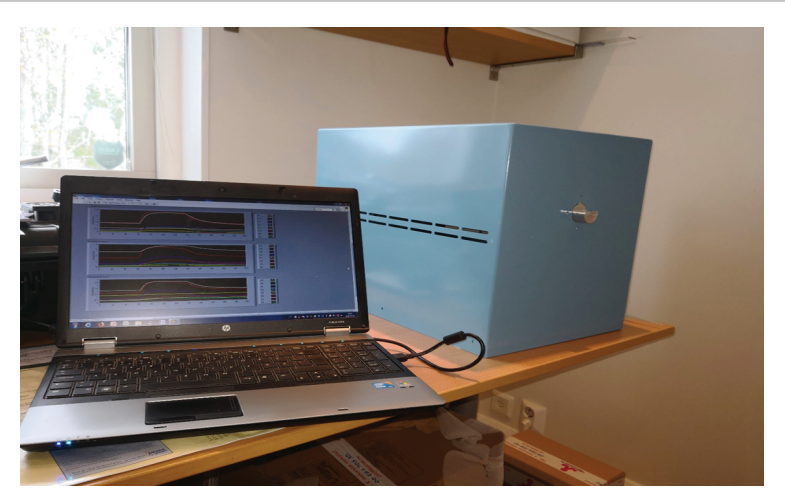

Figure 1: The first prototype, computer screen showing detected signals during the course of the assay.

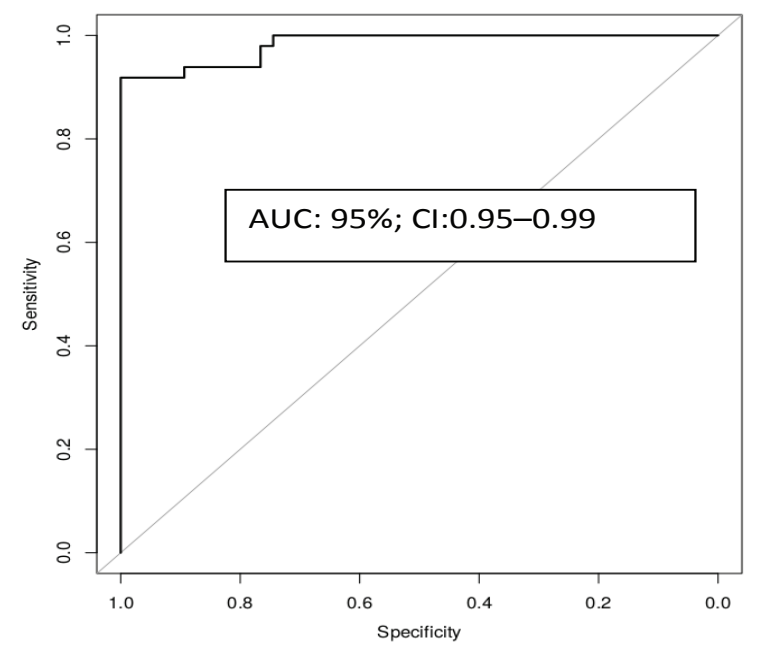

Figure 2: The ROC curve, the accuracy of the developed classification model was estimated in terms of ROC (receiver operating characteristic) curves, and the optimal sensitivity and specificity was derived from the ROC curve.

\section{Results}

\section{Gas chromatography}

Hydrocarbons predominated among the VOC in ovarian cancer samples. We identified 17 peaks both in the headspace of cancer and control tissues. Six of the 17 peaks had identical heights in both groups, and 11 were characteristic of cancerous tissue. Nine peaks occurred only in later groups.

The 11 peaks that were identified as characteristic for ovarian carcinoma were as follows:

1. Triethylamine.

2. Pyridine.

3. Toluene.

4. p-xylene.

5. E, Z-8,10-Dodecadien-1-ol.

6. Trifluoromethyl benzene.

7. Hexadecanoic acid.

8. Docosane.

9. 3-methylhexadecane.

10. Tetracosane.

\section{Heptadecane.}

The results of GC-MS analysis showed significant differences, namely, 10 out of 11 compounds (VOCs) present in cancer cells "headspace" were present either only in minimal amounts or not at all in control tissues. The exception was E, Z-8,10-dodecadien-1-ol, a pheromone that healthy tissues emit more than do cancer tissues. Whereas individual compounds of the list above have been shown to be markers of cancer, the panel has been shown to give an especially high statistical significance for ovarian cancer, compared with control tissues.

\section{Sensors}

Eight metal oxide semiconductor gas sensors $\left(\mathrm{SnO}_{2}\right)$ were built into each side of the detector tube, sensor positions called 1-8 (Figaro Engineering Inc., Japan). Selected sensors mainly detect different hydrocarbons and other VOCs. Each individual sensor can respond to several different substances, a function that can be controlled by changes in the sensors' heating voltage. The following sensors were built-in: TGS 2602, TGS 2603, TGS 2620, TGS 2611-E00, TGS 2600, TGS 2611 -C00, TGS 2444, and TGS 2610. At each position, an identical sensor was placed in each detector tube wall, giving a total of 32 sensors in the sensor tube. Since each sensor has a different heating voltage, i.e., it worked at a different temperature from that of its peers, there were varied signals from each sensor in a sensor set quadruple. The temperature differences enable four identical sensors to detect far more substances than four identical sensors working at the same temperature.

\section{Statistical analysis}

The results from the analysis total of 165 blood plasma samples, of which 97 were from patients with ovarian carcinoma and 68 from healthy controls, were first calculated externally by a medical statistician (JR), as described above. The area under the ROC curve was 0.9744 , and the 95\% CI was 0.9559-0.9929 (DeLong). With the threshold at 0.52 , the sensitivity was $92 \%$, and the specificity was $93 \%$ (Figure 2 ). 
Citation: Horvath G, Ranstam J, Ottosson M, Nilsen M (2018) New Construction of an Electronic Nose Detects Volatile Organic Compounds from Blood, useful for the Diagnosis and Screening of Ovarian Carcinoma. J Cancer Sci Ther 10: 387-391. doi: 10.4172/1948-5956.1000572

These results were later verified with automatic calculations programmed into the device. With automatic calculation, the device can show positive (cancer), and negative (not cancerous). Fifty samples from ovarian cancer patients and 50 from healthy controls were randomly selected from the same material. Their files, which were already stored in the unit's computer, were then run through the device's integrated statistical programme. Both the sensitivity and specificity were $92 \%$. These two coinciding analytic results strengthen the evidence that the device can reliably calculate the detected VOC signals and that it works in a stable way.

\section{Discussion}

We presented a developed form of an electronic nose for the diagnosis of ovarian cancer. The device is capable of detecting VOCs from $0.8-1.0 \mathrm{ml}$ of plasma from ovarian cancer patients, with $92 \%$ sensitivity and $93 \%$ specificity. Methods that have similar performance levels to the method described herein are not currently available for the diagnosis of this disease.

Ovarian cancer has a mortality rate within 5 years of slightly above $50 \%$. High mortality is primarily due to late diagnosis: Over $70 \%$ of newly diagnosed patients have Stage III or Stage IV. Several periods of delay, including patient and doctor delay, are significant contributing factors. The disease usually begins with insidious, diverse, and intermittent symptoms, sometimes for several years, and patients who seek primary care can often be investigated for other diseases [11]. Even if the doctor at the primary care stage thinks about ovarian cancer, s/ he has no available methods to verify it. Once the patient is referred to specialist clinics, even there, the available diagnostic methods such as ultrasound and tumour marker CA-125 have relatively low sensitivity and specificity, especially for the diagnosis of the early stages of the disease, although the sensitivity for the methods increases with tumour growth. Due to these shortcomings, these methods are less suited to the early diagnosis of ovarian cancer.

A practical use of the device presented here is a reliable, simple method to diagnose ovarian cancer with high sensitivity and specificity, simplifying the diagnosis of this disease even at the primary care level, while also allowing for a more accurate diagnosis at the specialist level. With safer opportunities for rapid diagnosis, many patients would receive treatment significantly earlier than they currently do. This, in turn, would improve survival rates. The fact that nearly half of the tested samples (40 of 97) from patients were from individuals with early stages of ovarian cancer strongly suggests that our device may be useful for early diagnosis.

Impartial external clinical trials have been started to validate the results from this study. In that study, detailed clinical and histopathological data will be analysed. The results will be able to provide more information regarding the practical use of the device within the existing health care system.

To our knowledge, this device is the first in the world to use blood (plasma) for the detection of VOCs emitted by cancer cells. The detection of these characteristic VOCs enables the device to be used for a reliable diagnosis of ovarian cancer. The concept that plasma contains significant quantities of VOCs from cancer had already been suggested from our previous experiments with trained dogs [3,4]. However, we were unable to verify the possible VOCs characteristic of ovarian cancer in previous experiments. As such, it was quite natural to then attempt to design an electronic nose that could manage the headspace of blood plasma with the required efficiency. Sensor selection was based on results from previous investigations carried out with gas chromatography. The results showed that hydrocarbons predominated among the VOCs of ovarian cancer. Aromatic hydrocarbons and other hydrocarbons may be derived from dead cells in the tumour mass. Up to $90 \%$ of the tumour mass may consist of dead or dying cells. Thus, these substances are present in much higher concentration in the blood of cancer patients than in the blood of healthy people. Central necrosis occurs early in tumours when they are just over $100 \mu \mathrm{m}$ in diameter [12]. Early detection of typical VOCs in ovarian cancer may be useful for early diagnosis of the disease.

We chose TGS metal oxide sensors $\left(\mathrm{SnO}_{2}\right)$ from Figaro Engineering Inc., Japan. These sensors can detect both aromatic and other hydrocarbons with good results. The sensors are relatively inexpensive and have long lifetimes. The use of plasma as a diagnostic material with the reported sensitivity and specificity signals promises great practical benefits for physicians and, not least, for patients.

The use of a device to screen for ovarian cancer is a more complex issue. Several serious studies have been done to test the possibilities of screening for ovarian cancer in this way. However, to date, none of these studies have been able to demonstrate survival benefits for patients who develop cancer. There are several weaknesses in the published studies, discussed in the medical literature as possible contributing factors to these failures $[1,13]$. Some authors also raised the issue of side-effects that can occur, primarily psychological ones, arising from studies using ultrasound and CA-125 detection [11].

However, our study-with demonstrated high sensitivity and specificity, including for early ovarian cancers - showed that this method may be a useful instrument for the screening of an apparently healthy population. Blood sampling done on an annual basis is unlikely to have negative psychological effects on the women involved. In addition, there are several other important aspects that need to be taken into account when designing a new study $[1,13]$. However, our data suggest that the screening is definitely worth further study.

\section{Conclusion}

In summary, we presented here development of the first clinically useful tool for the early diagnosis of ovarian carcinoma based on accurate detection of specific VOCs from plasma that are characteristic for ovarian carcinoma. Depending on the unit's simplicity, its high sensitivity suggests that in the future it can be an effective support tool for practical medical care worldwide. Presented work, however, is an initial, preliminary description of several years of development process that, with interesting results, opens both new issues and new opportunities. Several clinical, multicenter studies are needed in the future to be able to use the new instrument. Impartial external clinical trials have been started in Sweden to validate the results from this study. In that study, detailed clinical and histopathological data will be analysed. The results will be able to provide more information regarding the practical use of the device within the existing health care system.

\section{Declarations}

\section{Ethics approval and consent to participate}

This study was conducted in accordance with the regulations of the Helsinki Declaration (1964/2013). Permit for tumor banking and permit for study was approved by the Regional Ethics Committee, Gothenburg, Sahlgrenska Academy, University of Gothenburg (Ethical Committee license number: S-154-02 (for tumour bank,); Ethical Committee license number: S-220-08 (for tests and studies). 
Citation: Horvath G, Ranstam J, Ottosson M, Nilsen M (2018) New Construction of an Electronic Nose Detects Volatile Organic Compounds from Blood, useful for the Diagnosis and Screening of Ovarian Carcinoma. J Cancer Sci Ther 10: 387-391. doi: 10.4172/1948-5956.1000572

All samples used during the development period including samples for tests and studies were from the tumor bank. This study was conducted according to Uniform Requirements for Manuscripts Submitted to Biomedical Journals (URMS) recommendations.

\section{Consent for publication}

Not applicable

\section{Competing interests}

The authors declare no conflicts of interest.

\section{Funding}

Not applicable

\section{Author Contributions}

G. H.: Study initiator, patent owner, manuscript preparation, J.R.: Medical statistics, manuscript preparation, M.O., M.N.: Programming.

\section{Acknowledgements}

The authors would like to thank Johan Redeby, Royal Institute of Technology, Stockholm, Sweden for GC+MS analyse; Special thanks to the engineer Martin Brolin, Nacka It, Nacka, Stockholm for skilled help and good ideas; Mrs. Ghita Fallenius Vecchi and Mrs. Helena Kahu at the Research Laboratory, Department of Oncology, Sahlgrenska Univ. Hospital, Gothenburg, Sweden for their assistance.

\section{References}

1. Rauh-Hain JA, Krivak TC, Del Carmen MG, Olawaiye AB (2011) Ovarian cancer screening and early detection in the general population. Rev Obstet Gynecol 4: 15-21.
2. Horvath G, Jarverud GA, Jarverud S, Horvath I (2008) Human ovarian carcinomas detected by specific odour. Integr Cancer Ther 7: 76-80.

3. Horvath G, Andersson H, Poulsson G (2010) Characteristic odour in the blood reveals ovarian carcinoma. BMC Cancer 10: 643-647.

4. Horvath G, Andersson H, Nemes SZ (2013) Cancer odour in the blood of ovarian cancer patients: A retrospective study of detection by dogs during treatment, 3 and 6 months afterward. BMC Cancer 13: 396-399.

5. Labview 12 (2010) National Instruments 2: 1.

6. Tibshirani R (1996) Regression shrinkage and selection via the lasso. J R Statist Soc B 58: 267-288.

7. R Core Team (2012) A language and environment for statistical computing, Vienna, Austria.

8. Friedman J, Hastie T, Tibshirani R (2010) Regularization paths for generalized linear models via coordinate descent. J Stat Softw 33: 1-22.

9. Kuhn M, Wing J, Weston S, Williams A, Keefer C, et al. (2012) Classification and regression training. $R$ Package Version 5.15-044.

10. Robin X, Turck N, Hainard A, Tiberti N, Lisacek F, et al. (2012) pROC: An open-source package for $\mathrm{R}$ and $\mathrm{S}+$ to analyze and compare ROC curves. BMC Bioinformatics 12: 77-83.

11. Pepin K, Del-Carmen M, Brown A, Dizon DS (2014) CA 125 and epithelia ovarian cancer: Role in screening, diagnosis, and surveillance. Am J Hematol Onc 10: 22-29.

12. Sciumè G, Shelton S, Gray WG, Miller CT, Hussain F, et al. (2013) A multiphase model for three-dimensional tumor growth. New J Phys 15: 015005.

13. Jacobs IJ, Menon U, Ryan A, Gentry-Maharaj A, Burnell M, et al. (2016) Ovarian cancer screening and mortality in the UK collaborative trial of ovarian cancer screening (UKCTOCS): A randomised controlled trial. Lancet 387: 945-956. 